\title{
Phytoprotection
}

\section{Liste des participants}

phytoprotection

Volume 89, numéro 2-3, décembre 2008

Journée commémorative du centenaire de la société de protection des plantes du Québec

Centennial Commemoration of the Québec Society for the Protection

of Plants

URI : https://id.erudit.org/iderudit/038251ar

DOI : https://doi.org/10.7202/038251ar

Aller au sommaire du numéro

Éditeur(s)

Société de protection des plantes du Québec (SPPQ)

ISSN

0031-9511 (imprimé)

1710-1603 (numérique)

Découvrir la revue

Citer ce document

(2008). Liste des participants. Phytoprotection, 89(2-3), 171-173.

https://doi.org/10.7202/038251ar

Ce document est protégé par la loi sur le droit d'auteur. L’utilisation des services d'Érudit (y compris la reproduction) est assujettie à sa politique d'utilisation que vous pouvez consulter en ligne.

https://apropos.erudit.org/fr/usagers/politique-dutilisation/
Cet article est diffusé et préservé par Érudit.

Érudit est un consortium interuniversitaire sans but lucratif composé de l’Université de Montréal, l'Université Laval et l'Université du Québec à Montréal. Il a pour mission la promotion et la valorisation de la recherche. https://www.erudit.org/fr/ 


\section{Liste des participants}

\section{PHYTOPROTECTION $89:$ 171-173}

Alain Asselin, retraité du Département de phytologie, Université Laval

Gilbert Banville, retraité du ministère de l'Agriculture, des Pêcheries et de I'Alimentation du Québec (MAPAQ), membre SPPO

Micheline Boulanger, invitée

Sophie Banville, Institut des nutraceutiques et des aliments fonctionnels (INAF), Université Laval

Claude Tremblay, invité

Maryse Barrette, Department of Natural Resource Sciences, McGill University, membre étudiante SEO

Carole Beaulieu, Centre SĖVE, Université de Sherbrooke, membre SPPO

Guy Bélair, Centre de recherche et de développement en horticulture (CRDH), Agriculture et Agroalimentaire Canada, membre SPPO

Richard Bélanger, Département de phytologie, Centre de recherche en horticulture (CRH), Université Laval, membre SPPO

Robert Bellemare, Direction de l'environnement et de la protection des forêts, ministère des Ressources naturelles et de la Faune (MRNF)

Nicole Benhamou, Département de phytologie, $\mathrm{CRH}$, Université Laval

Diane Lyse Benoit, CRDH, Agriculture et Agroalimentaire Canada, membre SPPO

Danielle Bernier, Direction de I'innovation scientifique et technologique (MAPAQ), Laboratoire de diagnostic en phytoprotection, membre SPPO

Marie-Ėve Bérubé, Département de phytologie, Université Laval, membre étudiante SPPO

Josée Boisclair, Institut de recherche et de développement en agroenvironnement (IRDA), membre SEQ

Guy Boivin, CRDH, Agriculture et Agroalimentaire Canada, membre SPPO et SEQ

Simon Boudreault, Département de biologie, Université Laval, et Centre de foresterie des Laurentides (CFL), membre SEQ

Gaétan Bourgeois, CRDH, Agriculture et Agroalimentaire Canada, membre SPPO

Jean Denis Brisson, Service de la biodiversité et des maladies de la faune (MRNF), membre SPPO et SEO

Guy Bussières, Département des sciences du bois et de la forêt, Université Laval, membre SPPO

Odile Carisse, $\mathrm{CRDH}$, Agriculture et Agroalimentaire Canada, membre SPPO

Pierre Mathieu Charest, Département de phytologie, Université Laval

François Charrier, IRDA, membre SPPO

Conrad Cloutier, Département de biologie et CRH, Université Laval, membre SPPO et SEQ

André Comeau, Centre de recherche et de développement sur les sols et les grandes cultures (CRDSC), Agriculture et Agroalimentaire Canada, membre SPPO

Luc Couture, CRDSC, Agriculture et Agroalimentaire Canada, membre SPPO

Domingos de Oliveira, Département des sciences biologiques, Université du Québec à Montréal (UQAM), membre SEQ

Daniel Dostaler, Département de phytologie, Université Laval, membre SPPO et SEQ

Raymond-Marie Duchesne, Direction de l'agroenvironnement et du développement durable (MAPAQ), membre SPPQ et SEQ

Gilles Émond, retraité du MAPAQ, président du comité du centenaire SPPQ, membre honoraire SPPQ

Rachel Des Landes, invitée

Élaine Émond, invitée 
Patrick Émond, invité

Ralph H. Estey, Emeritus Professor, Department of Plant Science, McGill University, membre honoraire SPPO

Mario Fréchette, Direction de I'innovation scientifique et technologique (MAPAQ), Laboratoire de diagnostic en phytoprotection, membre SEO

Andrew Frève, MAPAQ, membre SPPO

Alain Garneau, Direction de l'innovation scientifique et technologique (MAPAQ), membre SPPO

Marie Garon, Direction de l'innovation scientifique et technologique (MAPAQ)

Gérard Gilbert, Direction de l'innovation scientifique et technologique (MAPAQ), Laboratoire de diagnostic en phytoprotection, membre SPPO

Gisèle Grandbois, présidente et chef de la direction, IRDA

Richard Hamelin, Ressources naturelles Canada, Service canadien des forêts, CFL, membre SPPO

Richard Hogue, IRDA, membre SPPO

Lida Zurini, invitée

Louise Innes, Service des relevés et des diagnostics (MRNF), membre SPPO

Christine Jean, travailleuse autonome, membre SPPO et SEQ

Andréa Jinek, Département de phytologie, Université Laval, et CFL, membre étudiante SPPQ

Caroline Labbé, Département de phytologie, $\mathrm{CRH}$, Université Laval

Geneviève Labrie, Centre de recherche sur les grains (CÉROM), membre SEO

Michel Lacroix, Direction de l'innovation scientifique et technologique (MAPAQ), membre SPPO

Gaston Laflamme, Ressources naturelles Canada, Service canadien des forêts, CFL, membre SPPO

Jean-Paul Laforest, doyen, Faculté des sciences de I'agriculture et de l'alimentation, Université Laval

Johanne Lafortune, Agence de réglementation de la lutte antiparasitaire de Santé Canada, membre SPPO et SEO

Jacques Laganière, retraité du MAPAO

Ginette Guilbault, invitée

Olivier Lalonde, Département de phytologie, Université Laval, membre étudiant SPPO et SEO

Maryse Leblanc, IRDA, membre SPPO

Geneviève Legault, Département de biologie, Université de Sherbrooke, membre étudiante SPPQ et SEQ

Anne Légère, Centre de recherche de Saskatoon, Agriculture et Agroalimentaire Canada, membre SPPO

Michel Letendre, retraité du MAPAQ et consultant en phytoprotection

André Lévesque, Centre de recherches de l'Est sur les céréales et les oléagineux, Agriculture et Agroalimentaire Canada, membre SPPO

Suzanne Marchand, Département de phytologie, Université Laval, membre SPPO

Carole Martinez, Département de phytologie, CRH, Université Laval, membre SPPO

Robin Morrall, professeur émérite, Department of Biology, University of Saskatchewan

Peterjürgen Neumann, professeur honoraire, Université de Montréal, membre honoraire SPPO

Guillemond Ouellette, chercheur émérite, Ressources naturelles Canada, Service canadien des forêts, CFL, membre honoraire SPPO

Carole Devin, invitée

Denis Pageau, CRDSC, Agriculture et Agroalimentaire Canada, membre SPPO

Vincent Philion, IRDA, membre SPPO

Édith Plante, IRDA, membre SPPQ

Stéphan Pouleur, CRDSC, Agriculture et Agroalimentaire Canada, membre SPPO

Claude Richard, retraité du CRDSC, Agriculture et Agroalimentaire Canada, membre honoraire SPPO

Geneviève Richard, IRDA, membre SPPO et SEO

Marc-André Richard, retraité du MAPAQ

Sylvie Rioux, Centre de recherche sur les grains (CÉROM), membre SPPO 
Danny Rioux, Ressources naturelles Canada, Service canadien des forêts, CFL, membre SPPQ Geneviève Roy, Premier Horticulture, membre SPPQ

Anne-Marie Simao-Beaunoir, Centre SĖVE, Université de Sherbrooke, membre SPPO

Marie Simard, Ressources naturelles Canada, Service canadien des forêts, CFL, membre SPPQ

Léon Tartier, retraité du MAPAQ, consultant PHYTOPROTECTION 2000, membre honoraire SPPQ

Lucette Tartier, PHYTOPROTECTION 2000, invitée

Vicky Toussaint, CRDH, Agriculture et Agroalimentaire Canada, membre SPPQ

Russell Tweddell, Département de phytologie, $\mathrm{CRH}$, Université Laval, membre SPPQ

Anne Vanasse, Département de phytologie, Université Laval, membre SPPQ

Luc Vézina, Direction régionale du Bas-Saint-Laurent, MAPAO 